\title{
Valoración de la elección de tratamiento conservador en la Enfermedad Renal Crónica
}

\author{
Ana Isabel Aguilera Flórez, Ana Cristina Alonso Rojo, Blanca Linares Fano, Sara Prieto Fidalgo, Lidia García \\ Martínez, Mario Prieto Velasco
}

Nefrología. Unidad de Diálisis. Complejo Asistencial Universitario de León. España

\section{Resumen}

Introducción: El tratamiento conservador es una opción de tratamiento en la enfermedad renal crónica. Esta elección es decisión del paciente y/o familia.

Objetivo: Analizar el proceso de elección de tratamiento conservador, identificar el perfil de paciente que lo elige, su supervivencia y quien comunica la elección.

Material y Método: Estudio observacional, retrospectivo. Se incluyeron pacientes que eligieron tratamiento conservador entre 2010-2017. Los datos se obtuvieron de los registros de enfermería en historia clínica. Se recogieron variables demográficas, supervivencia, Índice de Comorbilidad de Charlson, índice de Barthel y valores de los pacientes utilizando la herramienta Tarjetas de valores.

Resultados: Se estudiaron 95 pacientes: $41,05 \%$ hombres, edad media $82,36 \pm 9$ años, 27,37\% institucionalizados. La familia comunicó la elección en el $62,11 \%$ de los casos. La media del filtrado glomerular al inicio de la información fue $11,53 \pm 2,73 \mathrm{ml} / \mathrm{min}$, mediana del Charlson 8 (13-3), Barthel 55 (100-0) puntos. En el proceso de evidenciar valores, la tarjeta más elegida fue "personal sanitario responsable del tratamiento". La supervivencia media fue 496,19 días $\pm 553,8$. Viven menos los hombres y los institucionalizados, sin diferencia significativa. El riesgo de

Correspondencia:

Ana Isabel Aguilera Flórez

Complejo Asistencial Universitario de León

Nefrología. Consulta ERCA y Diálisis Peritoneal

C/Altos de Nava s/n. 24071 León

E-mail: aaguilera@saludcastillayleon.es muerte es mayor, al aumentar el Charlson y disminuir el filtrado glomerular $(p=0,01)$. La familia comunicó la elección de seguir tratamiento conservador en el $62,11 \%$ de los casos.

Conclusiones: El paciente que opta por tratamiento conservador es, una persona anciana, dependiente, con comorbilidades, supervivencia media en torno a 18 meses y en más de la mitad de los casos es la familia quien comunica la decisión de optar por ese tratamiento.

PALABRAS CLAVE: enfermedad renal crónica; tratamiento conservador; toma de decisión; supervivencia; información; preferencias del paciente.

\section{Assessment of the choice of conservative treatment in chronic kidney disease}

\section{Abstract}

Introduction: Conservative treatment is a treatment option in chronic kidney disease. The election of this treatment is determined by patient and / or the family.

Objective: The objective of the study was to analyze the process of choosing conservative treatment, identify the patient profile, the patient' survival and who communicate the election.

Material and Method: Observational, retrospective study. Patients who chose conservative treatment between 2010-2017 were included. The data were obtained from nursing records in clinical history. Demographic variables, survival, Charlson Comorbidity Index, Barthel Index and patient values were collected using the Values Cards tool. 
Resultados: The sample was 95 patients, $41.05 \%$ men, mean age $82.36 \pm 9$ years, $27.37 \%$ institutionalized. The family reported the choice in $62.11 \%$ of the cases. The mean glomerular filtration rate at the beginning of the information was $11.53 \pm 2.73 \mathrm{ml} / \mathrm{min}$, the median of Charlson index: 8 (13-3), median of Barthel index: 55 (100-0) points. In the process of contrasting values, the most chosen card was "health personnel responsible for treatment". The median survival was $496.19 \pm 553.8$ days. Men and the institutionalized live less, without significant difference. The risk of death is greater, as the Charlson index increases and the glomerular filtration rate decreases $(p=0.01)$.

The family announced the election to continue conservative treatment in $62.11 \%$ of cases.

Conclusions: Patients who opts for conservative treatment are elderly persons, dependents, with comorbidities, average survival of 18 months, and in more than half of the cases, the family is the one that communicates the decision to opt for that treatment.

KEYWORDS: chronic kidney disease; conservative treatment; decision making; surviva; information; patient preferences.

\section{Introducción}

La consulta de enfermedad renal crónica avanzada (ERCA) atiende a pacientes en estadío 4-5 ( $F G<30$ $\mathrm{ml} / \mathrm{min}$ ) de la enfermedad. En este momento, una de las intervenciones fundamentales va a ser preparar al paciente para el Tratamiento Sustitutivo Renal (TSR) ${ }^{1}$; poniendo especial énfasis en los cuidados pre diálisis, la educación y la información, que permitan al paciente, siempre que no haya contraindicaciones, una libre elección de la modalidad de TSR².

Esta libre elección de tratamiento aparece recogida en la Ley 41/2002, básica reguladora de la autonomía del paciente, que defiende su derecho a decidir libre y voluntariamente entre las opciones clínicas existentes, indicando así mismo la obligación de los profesionales de informar y, posteriormente, respetar esa decisión ${ }^{3}$. Así mismo, la Sociedad Española de Nefrología, dentro del proyecto Compromiso por la Calidad de las Sociedades Científicas en España, recomienda "No iniciar tratamiento sustitutivo renal con diálisis sin haber hecho previamente una toma de decisiones en la que participen el paciente, la familia y el médico ${ }^{\prime \prime 4}$.
La toma de decisiones fomenta la autonomía del paciente, garantizando el respeto a sus valores, preferencias y estilo de vida, poniendo a las personas en el centro de la atención sanitaria.

Con un lenguaje sencillo, materiales de apoyo y empleando preferiblemente Herramientas de Ayuda a la Toma de Decisión (HATD), el equipo multidisciplinar de la consulta ERCA, facilita información individualizada, ayudando al paciente a considerar cuidadosamente las opciones de tratamiento, a entender sus peculiaridades y el impacto que cada opción puede tener en su vida ${ }^{5}$.

El tratamiento conservador (TC) en la ERCA se puede definir como, la atención planificada, integral y centrada en el paciente, que integra los principios de los cuidados paliativos, con intervenciones para retrasar la progresión de la ERC y minimizar las complicaciones sin diálisis ${ }^{6}$. Es una de las opciones que, junto a la hemodiálisis, la diálisis peritoneal y el trasplante, se debe de ofertar a las personas con ERCA. La voluntad del paciente en primer lugar y/o su familia conjunta o subsidiariamente, la edad avanzada asociada a una elevada comorbilidad o el deterioro funcional importante, influyen en la elección de No iniciar tratamiento dialítico ${ }^{7}$.

Es importante que tanto el paciente como la familia, tengan la certeza de que su elección no supone una peor calidad asistencial, una menor calidad de vida ${ }^{8} y$ que existe la posibilidad de cambiar la modalidad de tratamiento elegida inicialmente. EI TC implica diseñar estrategias que garanticen esa calidad, y un adecuado acompañamiento del paciente hasta el final de sus días?.

Hay poca información disponible sobre la prevalencia de pacientes con ERC tratados con TC y los resultados son muy variables debido a la gran disparidad de la población analizada ${ }^{10}$. Conocer nuestros datos sobre la elección de TC facilitará una mejor organización de los cuidados que necesitan estas personas, así como poder ofrecer una atención integral, tanto al paciente como a su familia.

\section{Objetivo}

El objetivo de este estudio fue analizar el proceso de elección de TC en la consulta ERCA, identificando el perfil del paciente que lo elige, su supervivencia y quien comunica la elección. 


\section{Material y Método}

Se realizó un estudio observacional, retrospectivo. Los criterios de inclusión fueron: pacientes que habían pasado por el proceso de educación con HATD para la elección del TSR en la consulta ERCA del Complejo Asistencial Universitario de León, entre los años 2010-2017 y que optaron por TC, frente a las opciones de hemodiálisis o diálisis peritoneal. En nuestro hospital, siempre que es posible, los pacientes que inician TSR, pasan por el proceso de educación para la elección de la modalidad de tratamiento.

Se registraron variables demográficas, tiempo de supervivencia, Índice de Comorbilidad de Charlson Modificado por Edad (ICC), nivel de dependencia mediante el índice de Barthel, además de los valores/preferencias del paciente mediante la herramienta denominada tarjeta de valores. También se recogió la información sobre qué persona informaba al equipo de salud sobre la elección del TC. Los datos se obtuvieron de los registros de enfermería de la historia clínica.

Las tarjetas de valores son un material que se utiliza durante el proceso educativo con HATD, tienen por misión explorar los valores y preferencias el paciente; consta de 23 tarjetas que contienen diferentes mensajes (ver Tabla 1). Se les pide que las clasifiquen según el grado de importancia que tienen para ellos en: Muy importantes, Algo importantes o Nada importantes y que elijan las dos más importantes. Estas dos tarjetas están visibles durante el proceso de información y discusión, para que la elección de la modalidad de tratamiento pueda ser coherente con sus preferencias y su estilo de vida.

El tratamiento estadístico de los datos se realizó con el programa Stata 14. Se calcularon las medidas de tendencia central y dispersión en las variables cuantitativas, expresando la mediana, rango, media y desviación estándar. En las variables cualitativas se calcularon las frecuencias absolutas y relativas. Se empleo el $\mathrm{Chi}^{2}$ para comparación entre medias y el coeficiente de correlación de Pearson para establecer la asociación lineal entre variables cuantitativas. Se utilizó también la prueba de Mantel-Haenszel (Long-rak test) para comparar el número de fallecimientos esperados con el observado, y el análisis de supervivencia con el método kaplan-Meier y la razón de tasas.

Este estudio se ha realizado utilizando una base de datos existente en nuestra Unidad ERCA. No se han incluido datos de carácter personal que pudieran identificar a los pacientes de forma directa o indirecta, respetando
Tabla 1. Tarjetas de valores.

\begin{tabular}{l} 
TARJETAS DE VALORES \\
Autonomía \\
\hline Impacto para la familia \\
\hline Viajar \\
\hline Trabajar a media jornada \\
\hline Trabajar a jornada completa \\
\hline Personal sanitario responsable del tratamiento \\
\hline Tratamiento en hospital \\
\hline Tratamiento en casa \\
\hline Temor a estar solo \\
\hline Temor a la sangre o a los pinchazos \\
\hline Participar activamente en mi tratamiento \\
\hline Ambiente hospitalario \\
\hline Tratarse junto a otros pacientes \\
\hline Recibir material en casa \\
\hline Frecuencia de visitas al hospital \\
\hline Actividades de ocio \\
\hline Esquema fijo de tratamiento \\
\hline Ser independiente \\
\hline Tiempo de desplazamiento al centro de tratamiento \\
\hline Ser cuidado por otros \\
\hline Tiempo libre \\
\hline Libertad de horarios \\
\hline Utilizar agujas \\
\hline
\end{tabular}

los principios éticos y universales, así como, las normas internacionales de protección de datos y la legislación española vigente.

\section{Resultados}

Durante los años 2010-2017, pasaron por el proceso de educación con HATD en nuestra consulta ERCA 535 pacientes, de los cuales 95 (17,75\%) eligieron realizar tratamiento conservador. De estos, el $41,05 \% \quad(n=39)$ fueron hombres, con una edad media de $82,36 \pm 9$ años; $y$ el $58,95 \%(n=56)$ fueron mujeres con una edad media de $85,67 \pm 5,13$ años. La media de edad del total de la muestra fue de 84,32 años (rango entre 53 y 97 años), y la mediana de 85,57 años. En la Tabla 2 se muestran otras características sociodemográficas de la muestra a estudio.

La media del filtrado glomerular estimado al inicio de la información fue de $11,53 \pm 2,73 \mathrm{ml} / \mathrm{min}$, la mediana del ICC de 8 puntos (rango entre 13 y 3 puntos), y del índice 
Tabla 2. Variables sociodemográficas de la población a estudio $(\mathrm{N}=95)$.

\begin{tabular}{lcc}
\hline Variables & N & Porcentaje \\
\hline SEXo & & \\
Hombre & & \\
Mujer & 39 & $41,05 \%$ \\
TIPO POBLACIÓN DE RESIDENCIA & 56 & $58,95 \%$ \\
Rural & & \\
Urbano & 60 & $63,16 \%$ \\
INSTITUCIÓN & 35 & $36,84 \%$ \\
Si & & \\
No & 26 & $27,37 \%$ \\
QUIEN EXPRESA LA DECISIÓN & 69 & $72,63 \%$ \\
Familia & & \\
Paciente & & $62,11 \%$ \\
No Conocido & 59 & $28,42 \%$ \\
\hline
\end{tabular}

de Barthel de 55 puntos (rango entre 0 y 100 puntos) -dependencia severa-.

De todos los pacientes del estudio que realizaron el proceso educativo, se pudo utilizar la herramienta tarjetas de valores en 27 pacientes (28,42\%). Según el orden de preferencia, las cuatro tarjetas que obtuvieron un mayor porcentaje de elección fueron: "Personal sanitario responsable del tratamiento" el 6,32\% ( $n=12)$, "Ser independiente", "Impacto para la familia" y "Tratamiento en casa" el 3,68\% ( $n=7)$ cada una. La frecuencia de elección de las tarjetas se muestra en la Figura 1.

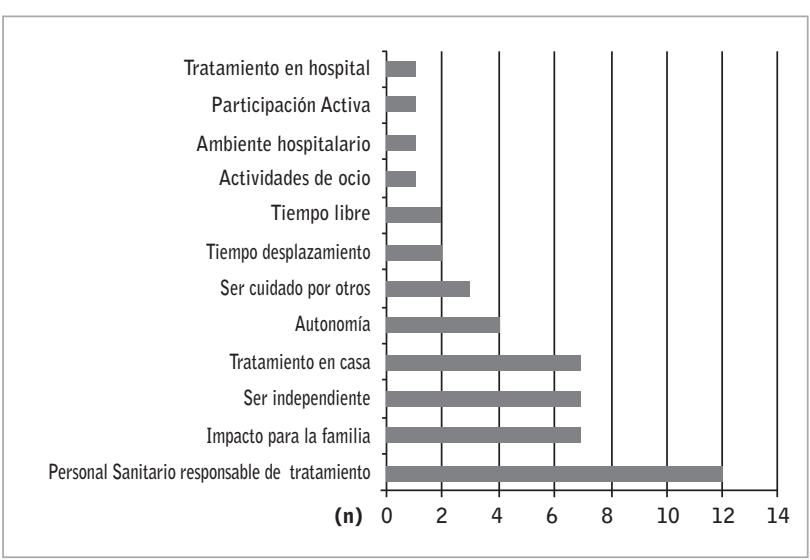

Figura 1. Distribución de tarjetas de Valores.

Al analizar el tiempo de supervivencia, observamos que la mitad de los pacientes fallecen antes de los 500 días tras manifestar su decisión de hacer TC, con un tiempo de vida medio de 496, 19 \pm 553 , 8 días y una mediana de 314 días, mientras que 13 pacientes permanecen vivos a los 2500 días, momento en el que finaliza este estudio (Figura 2).

Respecto a la supervivencia de los pacientes en función del sexo, como se puede apreciar en la Figura 2, los hombres viven menos que las mujeres, (Log-rank test $x^{2}=$ 3,29), siendo esta diferencia no estadísticamente significativa $(p=0,07)$. Es decir, el riesgo estimado de muerte en los hombres se multiplica por 1,52 respecto al de las mujeres $(H R=1,52$ IC95\% $=0,42-1,04)$.

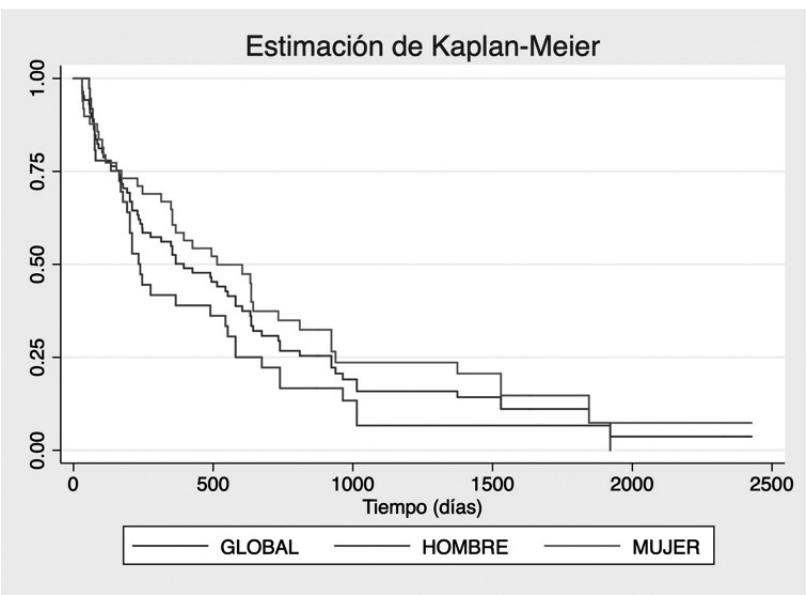

Figura 2. Análisis de supervivencia global y por sexo.

En cuanto al entorno, la supervivencia de los pacientes que viven en zona rural y urbana es muy similar (Logrank test $\left.X^{2}=0,09\right)$, siendo esta diferencia no estadísticamente significativa $(p=0,76)$. El riesgo de muerte en el medio urbano es tan solo 1,01 veces mayor que los que viven en el medio rural ( $\mathrm{HR}=1,01$ IC $95 \%=0,67-1,73$ ) (ver Figura 3).

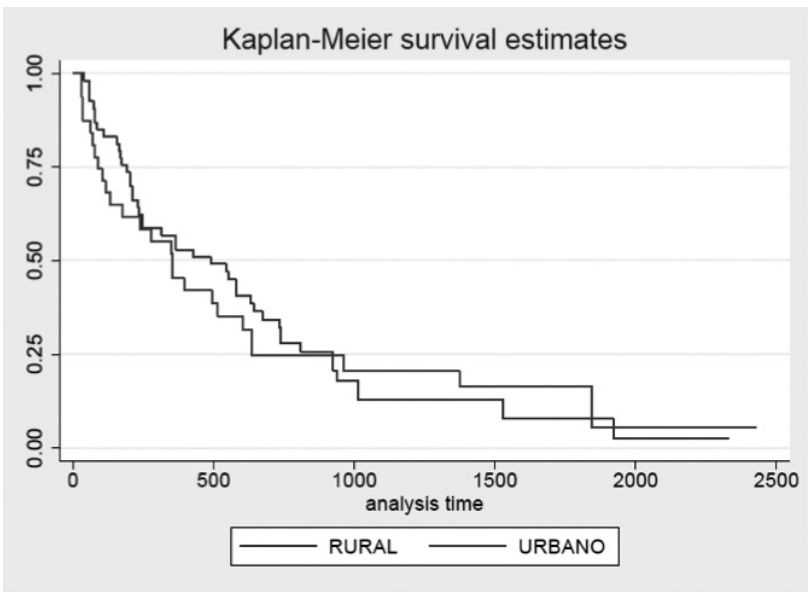

Figura 3. Análisis de supervivencia según tipo de población de residencia. 
Como podemos observar en la Figura 4, la supervivencia de los pacientes que viven en el domicilio familiar es mayor que la de los pacientes institucionalizados (Log-rank test $X^{2}=1,01$ ), siendo esta diferencia no estadísticamente significativa $(p=0,32)$. El riesgo de muerte es 1,32 veces mayor si el paciente está institucionalizado que si no lo está (HR=1,32 IC95\%=0,77-2,27).

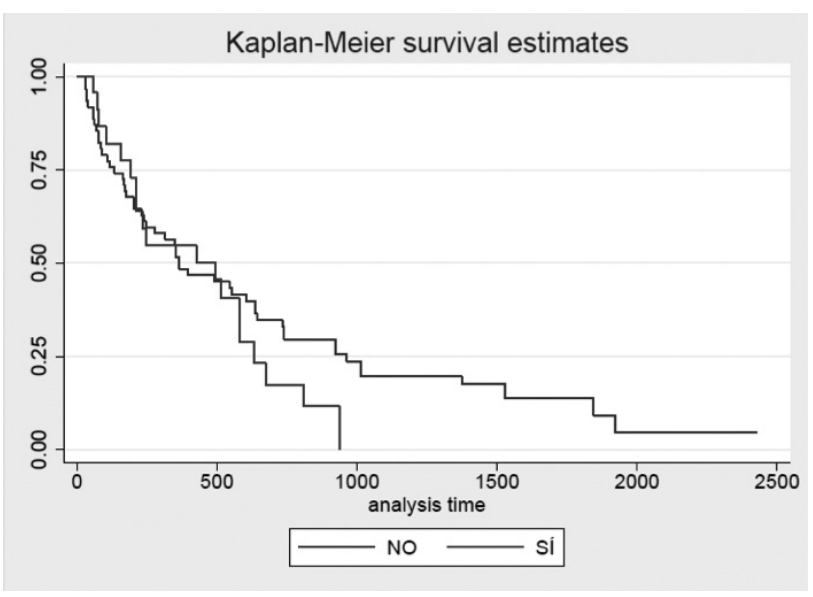

Figura 4. Análisis de supervivencia por institucionalización.

La edad a la que se realiza la información (84,32 años) y el ICC presentan una relación lineal directa débil, siendo esta significativa $(r=0,40, p<0,01)$; es decir, que a medida que la edad aumenta, el ICC también lo hace de forma significativa. El riesgo de muerte aumenta un $7 \%$ a medida que el ICC aumenta un punto, aunque no es estadísticamente significativo ( $H R=1,07$; IC $95 \%=0,92-1,22 ; p=0,39)$ y un $15 \%$ a medida que el filtrado glomerular disminuye un mililitro/min, siendo significativo. ( $H R=1,15 ; I C 95 \%=1,04-1,26 ; p=0,01$ ).

\section{Discusión}

Poco se sabe de cómo los adultos mayores con enfermedad renal crónica abordan las decisiones de tratamiento, o incluso cuántos optan por renunciar a la diálisis 5 .

En nuestro estudio la elección de tratamiento conservador fue del $17,75 \%$. Esta elección, se realizó después de pasar por un proceso educativo, en el que la provisión de información es un componente importante. La información precisa es fundamental, ya que se basa en conocer y comprender la mejor evidencia disponible sobre los riesgos y beneficios en todas las opciones disponibles, al tiempo que garantiza que los valores del paciente se tengan en cuenta y facilite una toma de decisión compartida ${ }^{6}$.

Este dato, está en consonancia con el publicado en diferentes estudios que muestran porcentajes, que varían entre el 18 y el $24 \%$ de elección de TC en personas mayores de 75 años $^{11-13}$.

Las personas que eligieron no realizar tratamiento de diálisis en nuestro estudio eran personas ancianas, con una dependencia severa para las actividades de la vida diaria y con una elevada comorbilidad. Este perfil coincide con el publicado en diferentes estudios, si bien varía la edad, que, en nuestra serie, con una mediana de 85,5 años, es superior a la edad de las series publicadas ${ }^{14-16}$.

La elección la expresó la familia en el $62 \%$ de los casos, mayormente, en aquellos con una dependencia y comorbilidad elevada. Y es que la familia, ejerce una gran influencia en la elección de la modalidad de tratamiento, máxime, cuando el paciente es dependiente ${ }^{17,18}$. La familia es imprescindible para ofrecer atención y apoyo a la persona enferma, estando directamente implicada en las circunstancias que rodean al tratamiento y evolución de la enfermedad ${ }^{19}$. Por ello, el soporte a los cuidadores es esencial, ya que, en la ERCA, los cuidadores suelen tener añadida una carga emocional que no es reconocible a primera vista ${ }^{20}$. Un trabajo realizado por enfermeras en el Reino Unido, con entrevistas a cuidadores familiares de pacientes sometidos a tratamiento conservador, expresaron preocupación y confusión, lo negativo del pronóstico y la incapacidad para hacerle frente; también consideraban el no tener esperanza y aceptar lo inevitable ${ }^{21}$.

En el proceso de evidenciar valores y preferencias, las tarjetas elegidas representan: la seguridad, la familia, la libertad y la preservación de su estilo de vida. Estos pueden considerarse, como los cuatro pilares que rigen la vida de las personas y que coinciden con los reflejados en la escasa bibliografía publicada ${ }^{22,23}$. Hay que resaltar, que el número de tarjetas recogidas es bajo, no siendo representativa del total de pacientes. Las razones por las que no están registradas, pueden ser diversas y al ser un estudio retrospectivo, desconocidas; lo que constituye una debilidad de este trabajo.

La mitad de nuestros pacientes viven alrededor de un año, este resultado es bajo con relación a otros estudios publicados, la causa podría ser, el momento en el que se ha realizado el corte del filtrado glomerular para medir la supervivencia. En nuestro estudio, la media fue $11,5 \mathrm{ml} /$ $\min$ frente a $15 \mathrm{ml} / \mathrm{min}$, de otras series publicadas ${ }^{12,24,25}$. 
Aunque también pueden intervenir otros factores, como la elevada comorbilidad o la edad, que disminuyen la esperanza de vida. En una revisión sistemática sobre el manejo conservador de la enfermedad renal en etapa terminal sin diálisis, se informa de una gran variabilidad de supervivencia con manejo conservador, entre 6,3 meses a 23,4 meses. El estudio que informó la supervivencia más corta, eran pacientes malos candidatos para diálisis, debido a múltiples comorbilidades o estado funcional alterado, factores que también disminuirían su expectativa de vida. Por el contrario, el estudio que informó la supervivencia más larga reclutó pacientes con la mediana de edad más joven ${ }^{13}$.

Llama la atención en nuestra serie, que las mujeres tienen mayor supervivencia, a pesar de tener una media de edad más elevada, y que tanto el ICC como el Barthel, son iguales en ambos sexos. La explicación la podemos encontrar en la literatura: en un estudio realizado en Reino Unido durante un periodo de 18 años, vieron que, en pacientes en tratamiento conservador, la edad $>75$ años y el sexo femenino, predijeron de forma independiente una mejor supervivencia ${ }^{12}$. Sería interesante haber valorado la tasa de progresión de la ERCA de ambos sexos, dato que no hemos estudiado.

En base a nuestros resultados podemos concluir que, en nuestra consulta, la proporción de pacientes que elige tratamiento conservador es de $17,75 \%$, el perfil del paciente que lo elige es una persona anciana, dependiente, con una comorbilidad elevada y preferentemente mujer. Es la familia quien expresa la decisión en más de la mitad de los casos y una cuarta parte de los pacientes en tratamiento conservador está institucionalizada. La supervivencia media es cercana al año y medio, si bien la mitad fallece durante el primer año.

Recibido: 25 noviembre 2018

Revisado: 11 diciembre 2018

Modificado: 19 diciembre 2018

Aceptado: 13 enero 2019

\section{Bibliografía}

1. Martínez-Castelao A, Górriz JL, Bover J, Segura-de la Morena J, Cebollada J, Escalada J et al. Documento de consenso para la detección y manejo de la enfermedad renal crónica. Nefrología 2014;34(2):243-62.

2. Martínez-0caña JC. Gestión de la enfermedad renal crónica avanzada. Dial Traspl 2011;32(4):165-7.

3. Ley $41 / 2002$, de 14 de noviembre, básica reguladora de la autonomía del paciente y de derechos y obligaciones en materia de información y documentación clínica. B0E» núm. 274, de 15 de noviembre de 2002:40126-32.

4. Ministerio de Sanidad, Servicios Sociales e Igualdad. Compromiso por la calidad de las Sociedades Científicas en España. [Acceso 25 julio 2018] Disponible en: http://10.15.5.20:8162/organizacion/ sns/planCalidadSNS/cal_sscc.htm

5. Ladin K, Weiner DE. Better informing older patients with kidney failure in an era of patient-centered care. Am J Kidney Dis. 2015;65(3):372-74.

6. Combs SA, Davison SN. Palliative and end-of-life care issues in chronic kidney disease. Curr Opin Support Palliat Care. 2015;9(1):14-9.

7. Prieto-Velasco M, Quiros P, Remon C; Spanish Group for the Implementation of a Shared Decision Making Process for RRT Choice with Patient Decision Aid Tools. The Concordance between Patients Renal Replacement Therapy Choice and Definitive Modality: Is It a Utopia? PLoS One. 2015 0ct 14;10(10):e0138811.

8. Tejedor A, De las Cuevas X. Cuidado paliativo en el paciente con enfermedad renal crónica avanzada (Grado 5), no susceptible de tratamiento dialítico. Nefrologia 2008; (Supl 3):S129-36.

9. Andreu L, Hidalgo MA, Moreno C. Tratamiento conservador ante la Enfermedad Renal Crónica. Enferm Nefrol 2015;18 (4):303-7.

10. Teruel JL, Burguera V, Gomis A, Rivera M, Fernández-Lucas M, Rodríguez $\mathrm{N}$ et al. Elección de tratamiento conservador en la enfermedad renal crónica. Nefrología 2015;35(3):273-9. 
11. $0^{\prime}$ Connor NR, Kumar P. Conservative management of end-stage renal disease without dialysis: a systematic review. J Palliat Med. 2012;15(2):228-35.

12. Chandna SM, da Silva-Gane, Marshall C, Warwicker $\mathrm{P}$, Greenwood RN, Farrington K. Survival of elderly patients with stage 5 CKD: Comparison of conservative management and renal replacement therapy. Nephrol Dial Transplant. 2011;26(5):1608-14.

13. Murtagh FE, Burns A, Moranne 0, Morton RL, Naicker S. Supportive Care: Comprehensive Conservative Care in End-Stage Kidney Disease. Clin J Am Soc Nephrol. 2016;11(10):1909-14.

14. Da Silva-Gane M, Wellsted $D$, Greenshields $H$, Norton S, Chandna SM, Farrington K. Quality of life and survival in patients with advanced kidney failure managed conservatively or by dialysis. Clin J Am Soc Nephrol. 2012;7(12):2002-9.

15. Morton RL, Turner RM, Howard K, Snelling P, Webster AC. Patients who plan for conservative care rather than dialysis: A National Observational Study in Australia. Am J Kidney Dis.2012;59(3):419-27.

16. Smith C, Da Silva-Gane M, Chandna S, Warwicker $P$, Greenwood R, Farrington K. Choosing not to dialyse: Evaluation of planned non-dialytic management in a cohort of patients with end-stage renal failure. Nephron. 2003;95(2):40-6.

17. Morton R L, Tong A, Howard K, Snelling P, Webster A C. The views of patients and carers in treatment decision making for chronic kidney disease: systematic review and thematic synthesis of qualitative studies. BMJ 2010; 340:c112.
18. Wuerth DB, Finkelstein SH, Schwetz 0 , Carey $H_{\text {, }}$ Kliger AS, Finkelstein FO. Patients' descriptions of specific factors leading to modality selection of chronic peritoneal dialysis or hemodialysis. Perit Dial Int 2002; 22(2):184-90.

19. Low J, Smith G, Burns A, Jones L. The impact of end-stage kidney disease (ESKD) on close persons: a literature review. NDT plus 2008;1(2):67-79.

20. Leiva JP, Sánchez R, García H, Fernández MJ, Heras $M$, Molina $A$ et al. Cuidados de soporte renal y cuidados paliativos. Nefrologia 2012;32(1):20-7.

21. Noble H, Kelly D, Hudson P. Experiences of carers supporting dying renal patients managed without dialysis. J Adv Nurs. 2013;69(8):1829-39.

22. Aguilera AI, Linares $B$, Alonso AC, Prieto M. Análisis del impacto de los valores personales en la elección del Tratamiento Sustitutivo Renal. Enferm Nefrol 2017;20(3):209-14.

23. Morton RL, Devitt J, Howard K, Anderson K, SneIling $P$, Cass A. Patient views about treatment of stage 5 CKD: a qualitative analysis of semistructured interviews. Am J Kidney Dis. 2010;55(3):431-40.

24. Murtagh FE, Marsh JE, Donohoe P, Ekbal NJ, Sheerin NS, Harris FE. Dialysis or not? A comparative survival study of patients over 75 years with chronic kidney disease stage 5. Nephrol Dial Transplant. 2007;22(7):1955-62.

25. Ellam T, El-Kossi M, Prasanth KC, El-Nahas M, Khwaja A. Conservatively managed patients with stage 5 chronic kidney disease- outcomes from a single center experience. QJM. 2009;102(8):54754.

Este artículo se distribuye bajo una Licencia Creative Commons Atribución-NoComercial 4.0 Internacional. https://creativecommons.org/licenses/by-nc/4.0/ 